\title{
Distúrbio de linguagem como parte de um transtorno global do desenvolvimento: descrição de um processo terapêutico fonoaudiológico
}

\author{
Language disorder in a pervasive developmental disorder: description \\ of a speech-language intervention process
}

\author{
Rubem Abrão da Silva ${ }^{1}$, Simone Aparecida Lopes-Herrera ${ }^{2}$, Luciana Paula Maximino De Vitto ${ }^{3}$
}

\begin{abstract}
RESUMO
O objetivo deste estudo de caso foi descrever a intervenção fonoaudiológica de uma criança autista, tendo sido este trabalho realizado na Clínica de Fonoaudiologia da Faculdade de Odontologia de Bauru (FOB-USP). A criança em questão era do gênero masculino, com diagnóstico de autismo realizado aos 24 meses de idade, e que, desde a época do diagnóstico, recebeu intervenção fonoaudiológica individual duas vezes por semana, sendo aqui relatados os primeiros nove meses de terapia. Os resultados mostram que a criança desenvolveu uma comunicação funcional por meio da linguagem oral e outras formas de simbolismo, além de contato ocular espontâneo e comunicativo e momentos de atenção conjunta com adultos e crianças.
\end{abstract}

Descritores: Transtornos globais do desenvolvimento infantil/terapia; Transtorno autístico; Transtornos do desenvolvimento da linguagem/terapia; Reabilitação dos transtornos da fala e da linguagem

\section{INTRODUÇÃO}

De acordo com o Manual Diagnóstico e Estatístico de Transtornos Mentais da Associação Americana de Psiquiatria ${ }^{(1)}$, a categoria transtornos globais do desenvolvimento (TGD) é referente aos transtornos que se caracterizam por prejuízos severos e invasivos em diversas áreas do desenvolvimento, como habilidades de interação social recíproca, habilidades de comunicação e presença de comportamentos, interesses e atividades estereotipados. Os prejuízos qualitativos que definem estas condições representam um desvio em relação ao nível de desenvolvimento do indivíduo, o que afeta sua adaptação social, educacional e de comunicação.

Trabalho realizado na Clínica de Fonoaudiologia da Faculdade de Odontologia de Bauru da Universidade de São Paulo - FOB-USP - Bauru (SP), Brasil.

(1) Aluno do Programa de Prática Profissionalizante em Linguagem Infantil da Pró-Reitoria de Cultura e Extensão da Universidade de São Paulo - USP, oferecido pela Clínica do Curso de Fonoaudiologia da Faculdade de Odontologia de Bauru - FOB-USP - Bauru (SP), Brasil.

(2) Doutora, Professora do Departamento de Fonoaudiologia da Faculdade de Odontologia de Bauru da Universidade de São Paulo - FOB-USP - Bauru (SP), Brasil.

(3) Doutora, Professora do Departamento de Fonoaudiologia da Faculdade de Odontologia de Bauru da Universidade de São Paulo - FOB-USP - Bauru (SP), Brasil.

Endereço para correspondência: Simone Aparecida Lopes-Herrera. Al. Octávio Pinheiro Brisolla, 9-75, Vila Universitária, Bauru - SP, CEP 17012-

101. E-mail: lopesimone@usp.br

Recebido em: 1/3/2007; Aceito em: 3/9/2007
Em geral, as alterações se manifestam nos primeiros anos de vida e podem aparecer associadas a alterações neurológicas ou quadros sindrômicos, variando em grau e intensidade de manifestações. Fazem parte desta categoria o transtorno autista, o transtorno de Asperger, transtorno de Rett e os transtornos desintegrativos da infância.

O autismo é considerado uma síndrome comportamental com etiologias múltiplas e curso de um distúrbio do desenvolvimento, sendo caracterizado por déficits de interação social, visualizado pela inabilidade na relação com o outro, usualmente combinado com déficits de linguagem e alterações de comportamento. No entanto, o quadro não envolve todas as áreas do comportamento na mesma proporção, sendo considerado causador de um prejuízo difuso ${ }^{(2-3)}$.

$\mathrm{O}$ autismo faria parte do chamado espectrum ou continuиm de distúrbios, que teria como problema central um prejuízo intrínseco no desenvolvimento da interação social recíproca e na linguagem, sendo que tais características variam na tipologia e na severidade com que se manifestam, sendo comum encontrar-se, na literatura da área, a utilização de termos como autismo de alto e baixo funcionamento para os quadros com menores e maiores alterações, respectivamente $^{(4-6)}$.

Os dados sobre a incidência do autismo tomam-se, muitas vezes, discrepantes, devido ao uso de critérios diagnósticos diversos. Há relatos de incidência de autismo em 7-16 casos para cada 10.000 crianças, sendo o autismo de alto 
funcionamento uma fração de 11 a $34 \%$ destes $\operatorname{casos}^{(7)}$; assim como há dados de que 1 em cada 150 crianças apresenta $\mathrm{TGD}^{(8)}$ ou de que a ocorrência é de até 208 casos para cada 100.000 pessoas (aproximadamente, 20 casos para cada 10.000 nascidos vivos) ${ }^{(9)}$.

É significativo notar que todas as descrições de crianças autistas incluem alterações importantes de linguagem, especialmente no que diz respeito a seu aspecto funcional. A questão da comunicação dessas crianças representa provavelmente o seu distúrbio mais importante e os estudos mais recentes a respeito da comunicação de crianças autistas e que fazem referência às questões relativas ao uso comunicativo da linguagem utilizam parâmetros baseados na teoria pragmáti$\mathrm{ca}^{(10-11)}$.

Há uma grande variedade de fundamentos teóricos, que pautam todas as abordagens terapêuticas com autistas, partindo de pressupostos distintos. Os enfoques e estratégias podem ser diversos, porém, os objetivos finais são os mesmos: melhorar as habilidades lingüísticas, sociais e $\operatorname{cognitivas}^{(5)}$.

Um dos pressupostos teóricos que podem ser utilizados na área da intervenção com linguagem é a Análise Comportamental Aplicada (ABA) ${ }^{(12)}$, que se baseia em princípios científicos do comportamento para construir repertórios socialmente relevantes e reduzir repertórios problemáticos. Nesta abordagem, comportamentos indesejados (como estereotipias e falta de atenção) podem ser retirados ou extintos e comportamentos desejados (como contato de olho e intenção comunicativa) podem ser reforçados e modelados por meio de estratégias como as que exigem: repetição, imitação, mandos, modelos, imitação, pareamento de estímulos, entre outras técnicas.

Foi realizado um estudo sobre programas comportamentais de intervenção, aplicados a crianças e jovens com autismo de alto funcionamento e síndrome de Asperger, desde a idade pré-escolar até a adolescência. Os resultados revelaram que crianças entre 3 e 5 anos (idade pré-escolar) com desenvolvimento normal apresentam, nesta fase, uma gama variada de habilidades de interação social, em decorrência dos estímulos a elas fornecidos, em contextos lúdicos de interação. Por estas razões, programas de desenvolvimento social e de comunicação para autistas, no período pré-escolar utilizam tipicamente as brincadeiras como contexto primário, sempre conduzidas por um adulto. Na realidade, o que estaria envolvido nestas brincadeiras seria a representação simbólica. As situações utilizadas poderiam envolver pequenos grupos e serem trabalhadas em "setting" fechado ou em ambiente natural. De forma geral, incluiriam o arranjo ambiental (organização do ambiente) para facilitar a participação e cooperação da criança, com cuidadosa seleção de materiais e atividades estruturadas para promover a participação verbal da criança ${ }^{(13)}$.

Neste sentido, este estudo de caso teve como objetivo descrever o processo de intervenção fonoaudiológica de uma criança com diagnóstico de autismo. Tal processo terapêutico utilizou os princípios da análise comportamental aplicada à linguagem aliados aos princípios da abordagem funcional da comunicação.

\section{APRESENTAÇÃO DO CASO CLINICO}

Foi realizado o estudo de caso de uma criança, do gênero masculino que, na época do diagnóstico fonoaudiológico, apresentava 24 meses de idade. Todos os procedimentos deste estudo de caso foram aprovados pela Comissão de Ética em Pesquisa (CEP) da Faculdade de Odontologia de Bauru, sob protocolo $\mathrm{n}^{\mathrm{o}} 115 / 2006$.

A mãe da criança procurou por ajuda fonoaudiológica, em uma Unidade Básica de Saúde (UBS), com a queixa de que seu filho não falava e era bastante agitado. Nesta mesma ocasião, a mãe foi orientada a procurar um médico neurologista e a criança foi também encaminhada para a Clínica de Fonoaudiologia da Faculdade de Odontologia de Bauru (FOBUSP), da Universidade de São Paulo.

Nesta ocasião, foram realizadas anamnese e avaliação fonoaudiológica. $\mathrm{Na}$ anamnese, não foram encontrados dados relevantes relacionados à concepção, gestação e parto. Com os dados fornecidos pela mãe e com a avaliação neurológica já realizada, pôde-se concluir que a criança não apresentava atraso no desenvolvimento neuropsicomotor. Não havia antecedentes familiares de transtornos psiquiátricos ou mentais, somente de que o pai da criança apresentou atraso de linguagem. A mãe informou também que a criança emitia apenas algumas palavras isoladas (/da/, /bo/ e /ke/) sem função comunicativa, apresentava atenção reduzida a objetos e demonstrava não compreender a linguagem oral. Revelou, ainda que a criança, quando desejava algum objeto, dirigiase ao outro por meio de toques, levando-o até o objeto desejado.

Para a avaliação da linguagem e dos aspectos do desenvolvimento cognitivo, foram utilizados o Protocolo de Observação Comportamental (PROC) ${ }^{(14)}$, assim como avaliação de linguagem em situações de interação espontânea e de atividades lúdicas. As sessões de avaliação foram gravadas em vídeo, para posterior análise. Durante a observação, foram oferecidos brinquedos à criança (miniatura de utensílios domésticos, animais, meios de transporte, blocos e peças para encaixe), sendo que a postura do avaliador foi a de estimular a criança a manipular os objetos. Os itens observados foram referentes às habilidades comunicativas, compreensão verbal e atividade simbólica.

Em relação às habilidades dialógicas, a criança não iniciava interação, não respondia ao interlocutor e não participava da atividade dialógica. Quanto à funcionalidade da linguagem, observou-se a presença da função instrumental, uma vez que a criança solicitava, por meio de um toque de mão, a ajuda do terapeuta para alcançar objetos. Também utilizou a função de protesto, quando o terapeuta retirava objetos de sua mão, propositalmente.

A criança respondeu assistematicamente ao seu nome, voltando-se para o interlocutor apenas nestas ocasiões. Em nenhum momento, aparentou compreender as solicitações do terapeuta, seja por comandos verbais ou gestuais. No entanto, foram observadas respostas comportamentais a sons de fala e ambientais, como procurar a fonte sonora após ruídos incidentais.

Durante a manipulação dos objetos propostos, a criança 
não apresentou organização ou funcionalidade, desistindo da atividade em alguns momentos e, em outros, utilizando o terapeuta como instrumento para superar algum obstáculo. $\mathrm{Na}$ avaliação da brincadeira simbólica, a criança fez uso convencional dos objetos, sem apresentar atividade simbólica com os mesmos, encontrando-se, na época da avaliação fonoaudiológica aqui relatada, no estágio sensório-motor do desenvolvimento cognitivo.

Também foi realizada a avaliação audiológica por meio de métodos comportamentais e eletrofisiológicos (com sedação), constatando-se a audição normal da criança.

O diagnóstico fonoaudiológico foi, ao final da avaliação fonoaudiológica, de distúrbio de linguagem como parte de um transtorno global do desenvolvimento (TGD), sendo o diagnóstico neurológico de transtorno autista.

Como conduta, após a avaliação fonoaudiológica, a criança foi encaminhada para terapia fonoaudiológica individual, duas vezes por semana, na própria clínica de Fonoaudiologia da FOB-USP e a família foi orientada a inserir a criança no ensino regular infantil e, também, em uma sala de estimulação de uma instituição especializada em autismo.

O processo terapêutico aqui relatado é o referente aos primeiros nove meses de intervenção fonoaudiológica, sendo que a criança ainda continua em terapia. De início, baseado nos dados de anamnese e avaliação fonoaudiológica e apoio na literatura específica da área, a meta terapêutica principal estabelecida foi a de desenvolver a compreensão das situações de comunicação e a intencionalidade da mesma, por meio do uso de habilidades comunicativas não-verbais e verbais, visando melhoria na inserção social, escolar e familiar da criança. Os objetivos específicos foram: desenvolvimento da atenção conjunta, do contato ocular, da capacidade de imitação, da atividade simbólica, além do estabelecimento de turnos interacionais e a atribuição de funcionalidade a objetos e situações comunicativas.

Em relação às estratégias adotadas no processo terapêutico, como pôde ser observado nos dados da avaliação fonoaudiológica, a criança apresentava maior tempo de atenção ao terapeuta e ao objeto de interação, quando o ambiente terapêutico possuía o menor número de estímulos dispersivos possível. Para isso, o ambiente de interação na terapia foi propositalmente rearranjado, de forma a se retirar todos os estímulos competitivos, como móveis e objetos de decoração, sendo o ambiente já filtrado composto apenas pela presença da criança, do terapeuta e de um único objeto de interação escolhido para cada sessão. O arranjo ambiental para direcionamento de atenção e obtenção de comportamentos atencionais adequados é uma das estratégias adotadas pela análise do comportamento e que pode ser aplicada à Fonoaudiologia. A criança foi instigada, de forma direcionada, a ter atenção a estímulos auditivos (de fala, musicais e ambientais), estímulos visuais (objeto de interação, movimentos do terapeuta, emissões e movimentos articulatórios), assim como imitar ações, vocalizações e movimentos articulatórios realizados pelo terapeuta.

Situações planejadas foram criadas para propiciar o aparecimento das habilidades comunicativas da criança, exigin- do-se da mesma intenção comunicativa, troca de turnos interacionais, assim como participação ativa nas interações (sendo estes considerados, neste momento, os comportamentos desejáveis). Utilizando-se os princípios da Análise Comportamental Aplicada, a criança foi recompensada, neste primeiro momento, por meio de reforços positivos sociais (como sorrisos, palmas, palavras de incentivo com entonação variada), quando realizava as atividades propostas ou quando apresentava algum dos comportamentos desejáveis, tornando a situação de interação agradável e gratificante.

$\mathrm{O}$ terapeuta realizava intervenções diretas, chamando a atenção da criança para ele de forma sistemática e dirigida sempre que esta tentava realizar alguma atividade de forma isolada ou auto-estimulatória, que não propiciasse interação e troca de experiências, sem a participação do terapeuta ou do objeto de interação.

Todas as ações realizadas, tanto pela criança como pelo terapeuta, eram acompanhadas de emissões do terapeuta que as representassem, em um processo de pareamento de estímulos, para que a criança associasse as emissões orais/verbais com os objetos ou ações que estas representavam e, também, estimulando a compreensão da linguagem oral e o desenvolvimento da capacidade de abstrair e simbolizar o código linguístico oral. Desta forma, foi trabalhada a atividade simbólica e a exploração funcional dos objetos conjuntamente.

Inicialmente, não foi objetivo primário da terapia, fazer com que a criança passasse a utilizar a linguagem oral como forma de comunicação, mas sim que ela compreendesse o principio básico da comunicação, que é seu caráter interacional. Esta foi, ao longo do tempo, percebida pela criança como uma forma de interação social, assim como um meio para expressar suas vontades e desejos. No entanto, foi preciso, inicialmente, fazer com que a criança interagisse de forma plena para assim ser dada maior ênfase aos diferentes níveis da linguagem. Desta forma, pode-se dizer que o nível de linguagem mais trabalhado neste primeiro momento foi o pragmático, sem deixar de estimular o desenvolvimento semântico-lexical. O nível fonético-fonológico será o enfoque terapêutico futuro, quando o paciente apresentar maior fluxo de fala.

Também foi realizado um trabalho junto à escola freqüentada pela criança, para que este local também fosse um ambiente estimulador de suas habilidades de comunicação. Para isso, foram dadas orientações a professores e educadores sobre condutas a serem tomadas frente a alguns comportamentos específicos, tais como: movimentos estereotipados e atividades monológicas, assim como formas de estimulação da comunicação e da linguagem.

A mãe e a família da criança receberam orientações do Setor de Psicologia da Clínica de Fonoaudiologia da FOBUSP, para que pudessem lidar da melhor maneira possível com as dificuldades encontradas ao longo do processo, assim como para que estes melhor visualizassem as evoluções obtidas.

No início do segundo semestre de intervenção, maior ênfase foi dada ao aspecto sintático da linguagem oral, uma vez que palavras isoladas passaram a ser utilizadas pela cri- 
ança para solicitar objetos, ações, nomeação e como forma de protesto. Neste sentido, nos momentos em que a criança se comunicava por meio de palavras isoladas, o terapeuta passou a exigir uma melhor elaboração frasal, dando o modelo e solicitando uma nova produção - o que, na análise comportamental aplicada, é chamado de estratégia de mando-modelo ou modelagem. Um estudo de base comportamental $^{(15)}$, que teve como objetivo avaliar a eficácia de procedimentos instrucionais, baseados na modelagem, no aumento da compreensão e expressão oral de duas crianças autistas em idade pré-escolar demonstrou que a intervenção com base na modelagem foi efetiva no aumento da compreensão de situações, que envolviam atividade simbólica e também, no aumento da produção de palavras e frases.

Em relação aos resultados do processo terapêutico fonoaudiológico, quanto ao desenvolvimento de atenção conjunta, em nove meses de terapia, o paciente aumentou significativamente seu tempo atenção conjunta nas atividades realizadas. Já após os quatro primeiros meses de terapia, não houve mais necessidade de continuar com as modificações ambientais realizadas anteriormente para evitar dispersões, sendo que os móveis foram reintroduzidos na sala de atendimento. Diversos objetos que possibilitavam interações foram levados à terapia ao mesmo tempo, possibilitando que a criança realizasse escolhas direcionadas sem dispersar a atenção. Nas atividades realizadas, mesmo com estímulos competitivos, a criança permaneceu atenta e participativa. Em alguns momentos, ainda era necessário que o terapeuta chamasse insistentemente a atenção da criança para uma atividade, uma vez que a mesma ainda tentava brincar de forma isolada, porém isto aconteceu em menos de $10 \%$ das situações propostas. Este dado contrasta com o encontrado na avaliação inicial da criança, na qual ela não direcionava a atenção a nenhum estímulo e brincava de forma isolada a maior parte do tempo.

Quanto ao desenvolvimento de contato ocular, a criança passou a utilizar com maior frequiência contatos oculares durante as interações com o terapeuta e familiares. Contatos oculares passaram de ocorrências circunstanciais, no início do processo, à busca de interação/ comunicação constante e espontânea, após nove meses de terapia. Os contatos oculares ocorreram juntamente com intenções comunicativas, por meio de turnos verbais, em momentos de nomeações, solicitações, assim como em momentos de protesto. Em atividades direcionadas, era possível manter um contato ocular ainda maior. Em algumas destas situações, a criança passou a compreender que a atividade só teria continuidade, caso o contato ocular ocorresse. Este dado, em comparação ao encontrado na primeira avaliação fonoaudiológica, na qual a criança não iniciava interação de forma espontânea, não respondia ao interlocutor e não participava de atividades dialógicas, demonstra a evolução da criança em relação a estes itens durante o processo terapêutico relatado.

Quanto ao desenvolvimento da intenção comunicativa, já após quatro meses de terapia, foram raros os momentos em que a criança utilizou o terapeuta como objeto. No início do processo terapêutico, como observado na avaliação inicial, a criança utilizava o adulto como objeto com freqüência e, por isso, durante todo o processo terapêutico, desde o início, estes comportamentos não mais foram aceitos pelo terapeuta; quando ocorriam, o terapeuta solicitava vocalizações e contato ocular da criança para efetivar a comunicação e fornecer alguma resposta (já que, quando a criança tendia a usar o adulto como objeto, era na intenção de solicitar algo).

Com a introdução de diversas atividades nas sessões terapêuticas, a criança teve a chance de demonstrar, de alguma forma, qual a brincadeira que seria realizada em determinado momento da sessão (introdução de alternativas para escolha). Isso foi feito por meio de gestos e emissões com significado, tais como /bóa/ para brincar com bola, /kao/ para brincar com carrinhos e onomatopéias de animais para a identificação de cada um deles. No inicio de cada sessão, o terapeuta passou a perguntar à criança qual seria a primeira brincadeira a ser realizada, tendo como respostas palavras com significado como as citadas acima. A atividade só era iniciada após a emissão de uma das palavras pela criança.

É importante destacar o papel das pausas/silêncio (respeito à troca de turnos) no processo dialógico. Como terapeutas, muitas vezes os fonoaudiólogos tendem a ocupar todo o espaço comunicativo, não fornecendo um tempo de espera pela resposta da criança. No processo aqui relatado, tendo por base a técnica de espera, tradicionalmente associada a procedimentos comportamentais, durante as interações, o terapeuta sempre aguardava a resposta da criança para que as intenções comunicativas desta pudessem aparecer com maior freqüência. Constantemente, a criança buscou interação com o terapeuta por meio de olhares, gestos e vocalizações, no momento em que percebeu a passividade do terapeuta na atividade (momento de espera do terapeuta).

Quanto à atividade simbólica, a evolução neste aspecto pôde ser constatada por meio de diversos comportamentos, tais como a utilização efetiva da linguagem oral para a comunicação, um significativo aumento lexical e desenvolvimento de brincadeiras simbólicas, sendo que estas eram ausentes na avaliação inicial. A criança passou a utilizar os objetos trabalhados de forma adequada, atribuindo a funcionalidade correta, além de emitir palavras isoladas com significado (/boa/ para bola, /mãw/ para mão, /kao/ para carro) e onomatopéias de diversos animais (cachorro, galo, cavalo, vaca, pato, gato) no contexto correto. Também foi possível observar a criança simulando atividades anteriormente realizadas, reproduzindo as situações.

Ao longo do processo terapêutico, as trocas de turnos interacionais e vocálicos desenvolveram-se significativamente. A criança compreendeu que a interação era necessária para que todas as atividades fossem realizadas. Isso pôde ser observado, uma vez que a criança passou a iniciar turnos, esperar respostas do interlocutor, assim como passou a manifestar prazer em realizar atividades onde a base era a troca de turnos (atividades dialógicas).

Já aos seis meses de terapia, diversas eram as situações em que a criança iniciava turnos, assim como respondia ao interlocutor, tais como: quando questionada sobre a atividade a ser realizada, solicitando objetos e atividades de forma verbal. 
Quanto ao fornecimento de funcionalidade aos objetos, durante as atividades realizadas, a criança utilizou todos os objetos com suas respectivas funcionalidades, desde os móveis da sala aos utensílios domésticos utilizados durante as atividades lúdicas. É importante ressaltar que, quando a criança desconhecia a funcionalidade de algum objeto, o terapeuta fornecia repetidas vezes o modelo, mostrando a funcionalidade à criança, que logo após reproduzia o esquema apresentado, utilizando-o posteriormente.

Os princípios da Analise Comportamental Aplicada destacam a importância da imitação para o aprendizado e estabelecimento de comportamentos adequados. Durante as atividades, pôde-se observar que a criança realizou muitas ações anteriormente feitas pelo terapeuta, ou ainda, quando a imitação era necessária para que uma atividade/ interação acontecesse, na maioria das vezes, a criança respondia de forma positiva. Muitas emissões feitas pela criança foram realizadas inicialmente, por meio de atividades em que a imitação era o ponto de partida. Onomatopéias, jogos vocálicos e desenhos realizados pelo terapeuta foram igualmente realizados pela criança em caráter de imitação, primeiramente, para só então adquirirem caráter intencional e funcional. A dificuldade em realizar comportamentos imitativos, muito comumente encontrada nas crianças autistas, por vezes impede a aquisição de comportamentos sociais mais complexos e, devido a isso, o desenvolvimento da capacidade de imitação deve ser um importante foco nos programas de intervenção precoce de base comportamental no autismo ${ }^{(16)}$, já que a habilidade de imitação tem efeito positivo no desenvolvimento da linguagem, da brincadeira simbólica e na atenção direcionada.

Ao longo de toda a intervenção, a criança demonstrou boa capacidade de generalização e desenvolvimento cognitivo satisfatório perante as metas terapêuticas. Condutas simbólicas foram utilizadas com grande freqüência pela criança, tais como: utilização de esquemas simbólicos para solicitar ou obter algum objeto fora de seu alcance, emissões com significado e representações simbólicas (como fingir estar dando comida para uma boneca).

Quanto à elaboração sintática, com seis meses de terapia, após obter maior uso da linguagem oral por parte da criança, o terapeuta começou a trabalhar a elaboração de frases. O terapeuta passou a solicitar produções com estruturas sintáticas mais elaboradas por parte do paciente. Isso foi feito nos momentos em que a criança fazia uso de palavras isoladas para solicitar objetos, ações e atividades. Nestas situações, após as emissões do paciente, o terapeuta fornecia o modelo adequado/ desejado, não atendendo suas solicitações e exigindo uma produção mais elaborada (estratégia de mando-modelo e espera) para que estas fossem atendidas. A criança, após algumas sessões de terapia, passou a ensaiar estas produções, inserindo novos elementos em sua fala. Após nove meses de terapia, em diversos momentos, a criança havia construído frases do tipo sujeito-verbo.

Em todo processo terapêutico que visa melhora da comunicação, a utilização dos resultados alcançados em terapia fora do ambiente terapêutico e em ambientes naturais da criança, é muito importante. Baseado em relatos da mãe da criança e da professora sobre seus comportamentos em casa e na escola, pôde-se concluir que a criança, após os nove meses iniciais de terapia fonoaudiológica, estava se relacionando de forma satisfatória com pessoas pertencentes à sua rotina, fora do ambiente clínico. Segundo a mãe, a comunicação já estava ocorrendo de forma verbal, na maioria das vezes, por meio de palavras isoladas (substantivos e verbos). A mãe relatava também que a criança estava muito mais calma e atenciosa às atividades por ela propostas. Seu desempenho na escola melhorou, já que aceitava ficar sozinho no ambiente escolar e apresentava um tempo de atenção significativo em atividades como desenho, pintura e colagem (anteriormente a criança só ficava na escola acompanhada de sua mãe, sendo dispensada em atividades que necessitassem maior concentração).

É importante relatar que a rede funcional em que a criança está inserida é favorável para um bom desenvolvimento, uma vez que o trabalho fonoaudiológico e escolar, assim como o acompanhamento psicológico da família vêm contribuir para alcançar os objetivos propostos.

\section{DISCUSSÃO}

A criança do presente estudo apresenta características de linguagem e comportamentos compatíveis com um quadro de autismo. Neste caso, o diagnóstico e intervenção precoce favoreceram a intervenção fonoaudiológica também precoce, o que por si só contribui para a evolução e o desenvolvimento de crianças com autismo ${ }^{(13)}$. Estudo realizado com crianças, que receberam diagnóstico de autismo aos dois anos de idade e que foram reavaliadas aos nove anos, mostrou que as que haviam sido submetidas a um maior número de horas de terapia fonoaudiológica entre os dois e três anos de idade eram mais eficazes no estabelecimento de comunicação funcional $^{(17)}$.

Os achados de anamnese e avaliação fonoaudiológica antes do início da intervenção mostraram que a criança apresentava um prejuízo no desenvolvimento das habilidades comunicativas, na atividade simbólica e compreensão da linguagem oral, o que permitiu a elaboração de um planejamento terapêutico fonoaudiológico voltado a estes déficits. Mais uma vez, nota-se a importância de uma boa avaliação/ observação da linguagem para nortear os princípios terapêuticos a serem adotados na prática fonoaudiológica ${ }^{(4,6)}$.

Embora a literatura nacional ${ }^{(5,11)}$ ressalte a importância do diagnóstico e intervenção fonoaudiológica precoce, nos transtornos globais do desenvolvimento, pouco é encontrado sobre metas e estratégias terapêuticas para estes transtornos. Desta forma, o trabalho aqui apresentado fornece sua contribuição à área, uma vez que estratégias terapêuticas foram descritas e, em casos semelhantes, poderão servir como apoio para outros planejamentos terapêuticos.

As habilidades simbólicas e de representação são consideradas pré-requisitos para a emergência do uso funcional da linguagem. Assim, as características de atraso e desvio de comunicação da criança autista podem ser atribuídas a falhas no desenvolvimento cognitivo ${ }^{(11)}$. A criança, foco do presente estudo, ao longo da intervenção fonoaudiológica, evo- 
luiu significativamente no brincar simbólico, assim como no uso funcional da linguagem, sendo que esta, ao final dos nove meses de intervenção, já estava sendo utilizada para solicitar objetos, ações, nomeação espontânea, assim como para indicar ações.

Quanto mais cedo se dá o desenvolvimento da linguagem nos quadros de autismo, melhor o prognóstico, sendo que quando esta não se desenvolve até os cinco anos, é indicativo de um atraso de linguagem significativo, sendo o prognóstico desfavorável ${ }^{(13)}$. Como a criança deste estudo de caso, ao final dos nove primeiros meses de intervenção, já estava desenvolvendo a linguagem oral, seu prognóstico poderia ser considerado favorável em termos de comunicação.

A criança em questão respondeu de forma satisfatória aos princípios da Analise Comportamental Aplicada $^{(12)}$ à área de linguagem, em conjunto a uma abordagem funcional da comunicação, adquirindo comportamentos como contato ocular, atenção conjunta e comunicativa, assim como a linguagem oral com função comunicativa interacional, de protesto, interativa, instrumental e de nomeação, por meio de estratégias típicas da análise comportamental aplicada, como a técnica de mando-modelo, tempo de espera, procedimentos de pareamento de estímulos, imitação e reforço positivo $^{(15-17)}$. Tais estratégias são extremamente facilitadoras no direcionamento de um processo terapêutico, muitas vezes considerado complexo por muitos fonoaudiólogos, como é o de crianças autistas que não apresentam comunicação oral quando chegam aos consultórios, clínicas ou instituições para iniciarem os atendimentos. Procedimentos comportamentais são de grande valia na instalação de comportamentos considerados adequados e desejáveis, como o estabelecimento de contato ocular, intenção comunicativa, atenção direcionada e aumento da produção oral - todos estes necessários à melhora na comunicação e interação social.

Estudo $^{(18)}$ que fez a comparação entre dois grupos de crianças autistas pequenas submetidas a 14 meses de um tipo de intervenção, um grupo a intervenção de base comportamental (que seguia princípios da análise comportamental aplicada) e outro de base eclética (que era uma combinação de métodos de terapia de integração sensorial e princípios de comunicação alternativa associados à estimulação de fala/ linguagem) demonstrou que, quando submetidas a testes cognitivos, motores, de linguagem e de comportamentos adaptativos, as crianças do grupo submetido à intervenção comportamental apresentaram melhores resultados em todas as provas, com diferenças estatisticamente significativas, com exceção dos testes de habilidades motoras.

Em relação à terapia fonoaudiológica no autismo, na década de 80 , os procedimentos de condicionamento operante, utilizados na análise comportamental aplicada, eram usados apenas com o objetivo de treinar gestos simbólicos em crianças autistas, favorecendo o treino na fala, porém apresentando resultado aquém do esperado nos demais componentes da linguagem e, desta forma, sendo vistos com reservas na prática fonoaudiológica ${ }^{(10)}$. Defendia-se que, em intervenções feitas em situações mais naturais, as quais a criança está exposta, a terapia abordaria situações de interação, facilitação, generalização, transferência e colaboração, dando um novo impulso às terapias fonoaudiológicas, diferentemente do que ocorria em situações muito direcionadas.

No entanto, nada impede que as situações naturais ${ }^{(16)}$ sejam trabalhadas em um contexto mais direcionado e com controle de comportamento, como foi demonstrado por este trabalho. Todos os aspectos da linguagem (fonéticofonológico, sintático, semântico e pragmático) podem ser trabalhados em situações naturais, contextualizadas, englobando aspectos como iniciativas de comunicação, atenção conjunta, contato ocular, atividade simbólica e desenvolvimento cognitivo, direcionados pelos princípios da Analise do Comportamento ${ }^{(18)}$, em prol do desenvolvimento da comunicação e da linguagem.

\section{COMENTÁRIOS FINAIS}

É evidente a importância de um trabalho fonoaudiológico individual precoce com crianças autistas, visando, por meio de metas terapêuticas adequadas, a interação social, atenção conjunta, troca de turnos interacionais e, conseqüentemente, aquisição da linguagem oral e compreensão do processo de comunicação.

As estratégias terapêuticas aqui apresentadas mostraramse eficazes para a criança em questão, uma vez que esta apresentou evolução frente ao processo terapêutico fonoaudiológico. Desta forma, mesmo em se tratando de um estudo de caso, nada impede que, em situações semelhantes, os princípios aqui utilizados, assim como as estratégias terapêuticas citadas, forneçam sua contribuição às intervenções na área fonoaudiológica.

\begin{abstract}
This study aims to describe language intervention process of an autistic child at the Speech Pathology Department at USP-Bauru. An autistic male child diagnosed at two years old was submitted to private language intervention twice a week, since diagnosis. The study reports the first nine months of therapy. Results show that the child developed functional communication through oral language and other ways of symbolism, in addition to spontaneous and communicative eye contact, moments of attention in activities with adults and children.
\end{abstract}

Keywords: Child development disorders, pervasive/therapy; Autistic disorder; Language development disorders/therapy; Rehabilitation of speech and language disorders 


\section{REFERÊNCIAS}

1. American Psychiatric Association - APA. Manual Diagnóstico e Estatístico de Transtornos Mentais - DSM-IV-TR. 4a ed. Porto Alegre: Artmed; 2002.

2. Gillberg C. Autism and pervasive developmental disorders. J Child Psychol Psychiatry. 1990;31(1):99-119. Review. Erratum in: J Child Psychol Psychiatry. 1991;32(1):213.

3. Schwartzman JS. Autismo Infantil. São Paulo: Memnon; 2003.

4. Pastorello LM. Síndrome de Asperger. In: Fernandes FDM, Pastorello LM, Scheuer CI. Fonoaudiologia em distúrbios psiquiátricos da infância. São Paulo: Lovise; 1996. p. 45-59.

5. Lopes-Herrera SA. Avaliação de estratégias para desenvolver habilidades comunicativas verbais em indivíduos com autismo de alto funcionamento e Síndrome de Asperger [tese]. São Carlos: Universidade Federal de São Carlos; 2004.

6. Perissinoto J. Diagnóstico de linguagem em crianças com transtornos do espectro autístico. In: Ferreira LP, Befi-Lopes DM, Limongi SCO, organizadores. Tratado de fonoaudiologia. São Paulo: Editora Roca; 2004. p. $933-40$.

7. Gillberg C. Asperger syndrome and high-functioning autism. Br J Psychiatry. 1998;172:200-9. Review.

8. Fombonne E. What is the prevalence of Asperger disorder? J Autism Dev Disord. 2001;31(3):363-4.

9. Dales L, Hammer SJ, Smith NJ. Time trends in autism and in MMR immunization coverage in California. JAMA. 2001; 285(9): 1183-5. Comment in: JAMA. 2001;285(22):2852-3.

10. Fernandes FDM. Aspectos funcionais da comunicação de crianças com síndrome autística [tese]. São Paulo: Faculdade de Filosofia, Letras e Ciências Humanas da Universidade de São Paulo; 1995.
11. Fernandes FDM. Terapia de linguagem em crianças com transtornos do espectro autístico. In: Ferreira LP, Befi-Lopes DM, Limongi SCO, organizadores. Tratado de fonoaudiologia. São Paulo: Editora Roca; 2004. p. $941-53$.

12. Cooper JO, Heron TE, Heward WL. Applied behavior analysis. Columbus: Merril Pub. Co.; 1989.

13. Paul R. Promoting social communication in high functioning individuals with autistic spectrum disorders. Child Adolesc Psychiatr Clin N Am. 2003;12(1):87-106; vi-vii.

14. Zorzi JL, Hage SRV. PROC - Protocolo de observação comportamental: avaliação de linguagem e aspectos cognitivos infantis. São José dos Campos: Pulso Editorial; c2004.

15. Drager KD, Portal VJ, Carrolus L, Castellano M, Gagliano C, Glynn J. The effect of aided language modeling on symbol comprehension and production in 2 preschoolers with autism. Am J Speech Lang Pathol. 2006;15(2):112-25.

16. Ingersoll B, Schreibman L. Teaching reciprocal imitation skills to young children with autism using a naturalistic behavioral approach: effects on language, pretend play, and joint attention. J Autism Dev Disord. 2006;36(4):487-505.

17. Turner LM, Stone WL, Pozdol SL, Coonrod EE. Follow up of children with autism spectrum disorders from age 2 to age 9. Autism. 2006;10(3):243-65.

18. Howard JS, Sparkman CR, Cohen HG, Green G, Stanislaw H. A comparison of intensive behavior analytic and eclectic treatments for young children with autism. Res Dev Disabil. 2005;26(4):359-83. 